Giulia Baselica

Università degli Studi di Torino

\title{
L'EQUIVALENZA IN TRADUZIONE: LA TEORIA DI KOMISSAROV E IL DIBATTITO NEI TRANSLATION STUDIES
}

\author{
Translation equivalence: \\ Komissarov's theory and the debate in Translation Studies
}

\begin{abstract}
The notion of 'equivalence' is at the center of the theoretical reflection on translation. The Soviet scholar Vilen Komissarov elaborated a theory of equivalence in translation, identifying five forms of equivalence corresponding to as many functions of the text object of translation (communicative, descriptive, syntactic, semantic, verbal).

In this article we propose a brief analysis of the results accomplished by the research carried out by Vilen Komissarov in the light of formulations expressed by exponents of Translation Studies and in the view of an ideal international dialogue dedicated to the topic of theory and practice of translation.

Keywords: Komissarov, Russian translatology, translation equivalence, translation studies, translation theory

\section{Riassunto}

Al centro della riflessione teorica sulla traduzione si pone la nozione di «equivalenza». Lo studioso sovietico Vilen Komissarov elaborò una teoria dell'equivalenza in traduzione, identificandovi cinque forme di equivalenza corrispondenti ad altrettante funzioni del testo oggetto di traduzione (comunicativa, descrittiva, sintattica, semantica, verbale).

Nel presente contributo si propone una rapida analisi degli esiti raggiunti dalle ricerche condotte da Vilen Komissarov alla luce delle formulazioni espresse dagli studiosi esponenti dei Translation Studies e nell'ottica di un'ideale dialogo internazionale dedicato al tema della teoria e della pratica della traduzione.

Parole chiave: equivalenza, Komissarov, teoria della traduzione, traduttologia russa, translation studies
\end{abstract}


Nel periodo compreso fra i primi anni Settanta e l'inizio degli anni Duemila il complesso, quanto controverso, concetto di equivalenza ${ }^{1}$ occupa un posto rilevante nella riflessione teorica intorno alla traduzione ${ }^{2}$ e nella produzione scientifica di Vilen Komissarov ${ }^{3}$.

${ }^{1}$ Il concetto di equivalenza è forse di per sé univocamente indefinibile: se in senso generale si può affermare che due elementi sono equivalenti quando rispetto a un determinato criterio essi hanno lo stesso valore e, di conseguenza, due proposizioni equivalenti hanno lo stesso valore di verità essendo quindi, nel ragionamento, reciprocamente sostituibili (Auroux, 1990: 826), in altri contesti, non caratterizzati dalla definitezza spaziale e temporale dell'atto dell'enunciazione, né dalla commensurabilità epistemologica, l'equivalenza che debba acquisire una connotazione di tipo funzionale sfugge a ogni designazione precisa. In questo caso, infatti, l'equivalenza identifica l'elemento che in una determinata situazione sia idoneo a soddisfare una certa esigenza funzionale. La selezione dell'elemento - non di rado appartenente a un repertorio più o meno vasto e composito - acquisisce un carattere soggettivo, in quanto il soggetto lo sceglie in base al grado di attenzione che egli accorda all'insieme degli elementi equivalenti presi in esame. Postulato di ogni possibile relazione di equivalenza è il principio secondo il quale un solo elemento può svolgere più funzioni e una sola funzione può essere assicurata da più elementi intercambiabili (Chazel, 1990: 826).

Esteso all'ambito della traduzione e, soprattutto, al dominio della traducibilità, come presupposto essenziale del processo traduttivo il concetto di equivalenza manifesta la sua multanime essenza e la sua feconda e mutevole indefinitezza, data dalla simultanea coesistenza di fattori condizionanti e speculari nelle due dimensioni, poste a confronto, del testo in lingua originale, e del testo in lingua d'arrivo, connessi con l'individualità, e l'autorialità, il contesto cronologico, spaziale e culturale.

2 D. Kelly pone in evidenza la molteplicità delle posizioni assunte dai teorici in merito al concetto di equivalenza. Se negli anni Sessanta alcuni studiosi in particolare, come Catford e Nida, definiscono l'equivalenza in termini di relazioni - e tale approccio verrà in seguito mantenuto da studiosi come Toury, Pym e Koller, fino alle soglie del secondo Millennio - altri linguisti - ad esempio Snell-Hornby e Gentzler - negano la nozione stessa di equivalenza, mentre altri, come Baker, considerano il concetto di equivalenza traduttiva fondamentalmente privo di status teoretico e tuttavia pragmaticamente utile al traduttore (Kelly, 2011).

3 Vilen Naumovič Komissarov (1924-2005) fu linguista, filologo, traduttore e, soprattutto, negli anni Sessanta e Settanta uno dei fondatori della teoria linguistica della traduzione. Pubblicò circa un centinaio di contributi, che comprendono importanti monografie dedicate alla teoria della traduzione, alla semasiologia, oltre che alla lingua inglese. Tra i titoli più noti è opportuno ricordare: Slovo o perevode (Discorso sulla traduzione, 1973); Lingvistika perevoda (Linguistica della traduzione, 1980), Teorija perevoda (Teoria della traduzione, 1990), Obščaja teorija perevoda (Teoria generale della traduzione, 1999), Sovremennoe perevodovedenie (Traduttologia contemporanea, 2000). 
Se la sintesi finale della riflessione teorica sulla traduzione - e quindi sulla equivalenza traduttiva - è compendiata nella monografia Sovremennoe pervodovedenie, pubblicata nel 2000 e riproposta in una riedizione postuma, apparsa nel 2007, del saggio Lingvistika perevoda, edito originariamente nel 1980, già nel 1968 Komissarov aveva citato il fenomeno, da lui definito «esclusivamente teorico», della non equivalenza lessicale (Komissarov, 1968: 6), in un breve saggio pubblicato sulla rivista Tetradi perevodčika ${ }^{4}$. In quello stesso fascicolo il linguista Aleksandr Rajchštejn aveva preso in esame il principio dell'equivalenza, dedicandovi, nel contributo intitolato $O$ perevode ustojčivych fraz, uno specifico paragrafo ${ }^{5}$. Il concetto di equivalenza traduttiva viene quindi introdotto nel dibattito sulla teoria e la pratica della traduzione con una marcata accezione concreta e la sua irrealizzazione è considerata una mera ipotesi teorica. Se due anni prima, sulle pagine della stessa rivista, i termini 'identità' ed 'equivalenza' sono impiegati indistintamente (Avramov, 1966: 103111) in un'ampia recensione del saggio Les problèmes théoriques de la traduction di Georges Mounin, nel 1965 il linguista britannico John Catford con il saggio A Linguistic Theory of Translation offriva forse una delle prime articolate ed esplicative trattazioni dell'idea di equivalenza.

Catford opera una prima distinzione tra equivalenza traduttiva - in quanto fenomeno empirico rilevato dalla comparazione tra testi in lingua di partenza e relative versioni in lingua d'arrivo - e condizioni di equivalenza. L'equivalenza traduttiva, inoltre, si manifesta come equivalenza testuale (l'equivalente testuale è un testo o un segmento di testo in lingua d'arrivo ritenuto equivalente a un dato testo o segmento di testo in lingua di partenza) sia come corrispondenza formale (un corrispondente formale è, invece, una categoria della lingua d'arrivo - unità, classe o struttura - che si ritiene occupi, il più esattamente possibile, lo stesso posto, nell'economia della lingua d'arrivo, occupato da quella determinata categoria nella lingua di partenza). Questa è - precisa Catford

${ }^{4}$ Il periodico Tetradi perevodčika (I quaderni del traduttore), fondato nel 1958 dal linguista Viktor Rozenberg, diventò, a partire dal 1963, l'organo di pubblicazione della cattedra di Teoria e pratica della traduzione presso l'Istituto di Lingue Straniere Thorez di Mosca. La rivista divenne la sede di un fecondo dibattito teorico-pratico su questioni di teoria generale della traduzione, su specifici aspetti teorico-pratici, sulle più varie metodologie didattiche della traduzione, sui problemi inerenti alla valutazione della qualità delle traduzioni. La pubblicazione della rivista cessò nel 1989, ma tuttora essa costituisce una importante fonte storiografica per la ricostruzione del pensiero sovietico e russo sulla traduzione.

${ }^{5}$ Lo studioso si sofferma sulle possibili modalità traduttive di fraseologismi e forme fisse dal tedesco al russo individuando alcune specifiche categorie di traducenti equivalenti: totali, parziali, contestuali e situazionali, prestiti (Rajchštejn, 1968: 29-43). 
- quasi sempre approssimativa, in quanto ogni lingua è sui generis e le categorie che la costituiscono sono definite in termini di relazioni atte a garantire l'unità, la stabilità e la coesione della lingua stessa ${ }^{6}$.

Le condizioni di equivalenza attengono all'insieme dei tratti situazionali contestualmente rilevanti - i quali orientano la selezione di una determinata forma linguistica ritenuta equivalente - assegnati da Catford alla categoria della sostanza situazionale.

Allo studio dell'equivalenza, tuttavia, il linguista statunitense Eugene Nida aveva contribuito con un corposo saggio, Towards a Science of Translating: With special Reference to Principles and Procedures Involved in Bible Translating, accolto nel dibattito sovietico in anni successivi alla pubblicazione del manuale di John Catford. Laccertata inesistenza di equivalenti identici costituisce il presupposto della teoria elaborata da Nida; ne deriva che, osserva lo studioso, nell'atto della traduzione è necessario tentare di individuare l'equivalente più prossimo possibile all'elemento considerato del testo originale. Due sono i tipi di equivalenza: formale e dinamica. La prima pone attenzione al messaggio in sé, nella duplice componente di forma e contenuto, realizzandosi nella corrispondenza fra poesia e poesia, frase e frase, concetto e concetto. In tale prospettiva, appunto formale, il messaggio espresso nella lingua di arrivo dovrebbe il più possibile riprodurre i vari costituenti del messaggio. La seconda si fonda sul principio dell'«effetto equivalente»o «risposta simile», concetto discusso da Rieu e Phillips nel 1955 (Rieu, Phillips, 1955) 7.

La traduzione caratterizzata dall'equivalenza dinamica, precisa Nida, mira a produrre un'espressione del tutto naturale e a correlare il ricettore del testo tradotto con le modalità comportamentali proprie al contesto culturale al quale il lettore appartiene. Nida evidenzia tre diversi tipi di equivalenza dinamica connessi con altrettante situazioni contrastive: in presenza di lingue e culture reciprocamente affini; culture affini e lingue dissimili; lingue e culture dissimili ${ }^{8}$.

${ }^{6}$ Secondo lo studioso sarebbe possibile stabilire una vera e propria norma traduttiva a partire da una disamina quantitativa di occorrenze: calcolando il numero di occorrenze per ogni equivalente, dividendo tale cifra per il numero totale dell'elemento considerato nel testo fonte, si ottiene il valore delle probabilità dell'equivalenza (Catford, 1965).

7 Il principio dell'effetto equivalente era stato oggetto di indagine negli anni Quaranta negli studi prodotti dalla Scuola di Praga: per esempio in V. Procházka (Procházka, 1942).

${ }^{8}$ Se le lingue poste a confronto dal processo traduttivo sono legate da forte affinità, al traduttore si presenta l'elevato rischio di incorrere nell'errore di riconoscervi un'illusoria, superficiale, nonché falsa somiglianza; se, al contrario, esse si differenziano notevolmente, ma le culture di cui sono espressione sono affini, il traduttore dispone di ampi mezzi espressivi (Nida, 1964). 
La traduzione formalmente equivalente è, nell'essenza, una traduzione semantica (o source-oriented) che mira a rivelare quanto più possibile sia della forma sia del contenuto del testo originale. La traduzione dinamicamente equivalente è invece incentrata sulla risposta del ricevente e realizza l'equivalente naturale più prossimo al messaggio espresso nella lingua di partenza ${ }^{9}$. La connotazione di naturalezza attribuita alla concreta realizzazione dell'equivalenza attiene, sottolinea lo studioso americano, a tre componenti del processo comunicativo: alla lingua e cultura del lettore-ricevente; al contesto del messaggio; al pubblico della lingua e della cultura di arrivo.

La naturalezza espressiva nella lingua d'arrivo è, nell'essenza, una questione di co-rispondenza, co-suitability (Nida, 1964: 168). Essa deve inoltre considerare elementi altri, come l'intonazione, il ritmo della frase, il tono emotivo ${ }^{10}$.

Del saggio di Eugene Nida nel 1971 viene pubblicata in «Tetradi perevodčika» una meticolosa recensione, Na puti $k$ sozdaniju nauki o perevode, firmata proprio da Vilen Komissarov. Il linguista sovietico elogia l'intento multidisciplinare dell'approccio proposto da Nida e giudica utile e fecondo il dialogo fra la linguistica, l'antropologia e la psicologia, tuttavia rileva alcune sostanziali criticità nella determinazione del processo traduttivo finalizzato alla realizzazione dell'equivalenza. Komissarov osserva, per esempio, che nell'opera di Nida non viene descritto, né, quindi, motivato, il procedimento mediante il quale il traduttore seleziona i traducenti, stilisticamente e semanticamente equivalenti e tuttavia non di rado distanti dalla forma dei corrispondenti originali.

Tuttavia nel suo già menzionato studio Lingvistika perevoda ${ }^{11}$ Vilem Komissarov esamina più approfonditamente la trattazione dell'equivalenza traduttiva esposta da Nida, scardinando gli elementi portanti della teoria stessa: la traduzione, e quindi equivalenza, formale; e la traduzione, e quindi

9 Alla fine degli anni Settanta Werner Koller dette un rilevante contributo allo studio del fenomeno dell'equivalenza traduttiva (Koller, 1979), operando una distinzione fra le nozioni di 'corrispondenza' (l'esito della comparazione fra due sistemi linguistici sulla base del parametro saussuriano di langue e degli indizi di interferenze lessicali, morfologiche e sintattiche) ed 'equivalenza' (realizzata concretamente in elementi equivalenti rilevati nel confronto fra due sistemi linguistici, sulla base del parametro saussuriano della parole). Koller individua cinque tipi di equivalenza: denotativa, connotativa, testuale, pragmatica, formale.

${ }^{10} \mathrm{Nida}$ individua poi le zone di tensione fra equivalenza formale ed equivalenza dinamica, presenta ed esemplifica le limitazioni poste all'equivalenza dinamica e si sofferma sui condizionamenti traduttivi derivanti dalla tradizione.

${ }^{11}$ La cui prima edizione apparve nel 1980, quindi otto anni dopo la pubblicazione in «Tetradi perevodčika» della recensione al saggio di Nida e sedici anni dopo l'edizione originaria di quest'ultimo. 
equivalenza, dinamica. Nei due rispettivi aggettivi, 'formale' e 'dinamica' lo studioso sovietico coglie innanzi tutto una valenza marcatamente valutativa, per sua natura almeno in parte determinata dall'approccio soggettivo del traduttore del testo oggetto di traduzione. Nella stessa definizione di «equivalenza formale» individua una fondamentale contraddizione: essa identifica, nella sostanza, una traduzione essenzialmente letterale, alla quale, precisa Komissarov riprendendo Nida, sarebbero sottoposte anche le locuzioni idiomatiche, così contravvenendo alla norma della naturalezza, che costituisce un requisito essenziale per una traduzione comunicativamente di uguale valore, ravnocennyj - quindi equivalente - all'originale. Proprio tale connotazione assiologica rappresenta per Komissarov un autentico presupposto ontologico all'esistenza stessa della traduzione di ogni testo, in quanto fatto comunicativo unico, che presenta una struttura comunicativa dotata di senso e si caratterizza - oltre che per l'orientabilità situazionale e la selettività della capacità descrittiva della situazione - anche per la funzionalità comunicativa.

Così il concetto di «equivalenza dinamica» si rivela contraddittorio: Komissarov osserva che gli esempi proposti da Nida evidenziano la necessità di apportare alcune modifiche strutturali all'enunciato in lingua d'arrivo, al fine di trasferire completamente il contenuto del testo originale e, in tal modo, il requisito dell'effetto equivalente si riduce alla realizzazione della condizione di massima prossimità possibile fra la percezione del testo da parte del lettore dell'originale e la percezione del testo da parte del lettore della traduzione, il che non equivale, conclude Komissarov, a determinare un certo tipo di comportamento, cioè una «risposta simile ${ }^{12}$ (Komissarov, 1971).

Per Komissarov l'equivalenza traduttiva è un fatto reale, che è possibile osservare, descrivere e valutare. Il grado di prossimità fra l'originale e la

12 Precisando che le obiettive difficoltà con le quali si confronta ogni tentativo di imporre alla traduzione il doveroso obbligo di mantenere, e quindi di trasferire, la totalità del contenuto nel testo in lingua d'arrivo derivano dall'essenza stessa della comunicazione interlinguistica, Komissarov evoca la «presunzione» di equivalenza che spontaneamente il lettore della traduzione attribuisce alla traduzione. Analogamente Nida, esplicitando, nella realizzazione dell'equivalenza dinamica le difficoltà di resa lessicale nel caso in cui le lingue poste a confronto nel processo di traduzione siano espressione di culture reciprocamente distanti, sottolinea la naturale consapevolezza, propria di ogni popolo, della specificità culturale e la conseguente, altrettanto spontanea accettazione di un'irrealizzabile assoluta naturalizzazione. Entrambi gli studiosi accennano, seppur in prospettive opposte, - con riferimento nel primo caso alla presunzione di equivalenza, e nel secondo alla presunzione di non equivalenza - al ruolo nodale svolto dal ricettore della traduzione, il quale decreta, in ultima analisi, la riuscita o il fallimento della traduzione come processo comunicativo interlinguistico e interculturale 
traduzione è tuttavia determinabile in ogni singolo caso, in base a svariati fattori - oggettivi e soggettivi - e non può quindi essere univocamente prestabilito e imposto a ogni tipo di traduzione. Secondo il linguista sovietico la nozione di equivalenza non ha carattere normativo ed esprime, così, l'essenza della natura descrittiva della teoria della traduzione ${ }^{13}$ : nello studio di tale nozione egli identifica uno dei principali compiti della linguistica della traduzione, poiché la preminente importanza della massima corrispondenza fra il contenuto del testo originale e il contenuto del testo tradotto appare del tutto evidente. Di qui una prima serie di conclusioni indicate dallo stesso Komissarov. In primo luogo la condizione di equivalenza comporta una specifica definizione di traduzione come "sostituzione di materiale testuale in una lingua (LP) con materiale testuale equivalente in un'altra lingua (LA) (Komissarov, 1971). In secondo luogo il concetto di equivalenza acquisisce carattere qualitativo e valutativo: una traduzione corretta è, infatti, essenzialmente, una traduzione equivalente: è proprio l'equivalenza all'originale a conferire a un testo lo status di traduzione ${ }^{14}$. Infine, se l'equivalenza costituisce la condizione ontologica della traduzione stessa, il compito del linguista consiste nel determinare tale condizione nel modo più preciso possibile, innanzi tutto definendo ed esplicitando la nozione di equivalenza, quindi indicando gli elementi che nell'atto traduttivo devono essere conservati, cioè trasferiti nel testo tradotto.

Sulla base del presupposto secondo il quale il grado di prossimità semantica tra originale e traduzione rappresenta una grandezza variabile, Komissarov individua cinque tipi di relazioni di equivalenza, nei quali rientrano anche relazioni negative.

Il primo tipo contempla le traduzioni in cui la corrispondenza di contenuto con i relativi testi originali è inferiore rispetto allo stesso parametro misurato nelle traduzioni considerate invece equivalenti ${ }^{15}$. Tali traduzioni, osserva lo studioso, non trasmettono - o trasmettono solo parzialmente - il contenuto diretto e le unità lessicali e grammaticali del testo fonte, bensì riproducono

13 Natura dichiarata, se non addirittura propugnata nella concezione teoretica di James Holmes e nell'atto di nascita dei Translation Studies (Holmes, 1988).

14 Komissarov precisa inoltre che l'assenza di equivalenza indica che il testo considerato non è una traduzione, $o$, comunque è una traduzione non esatta e non adeguata (Komissarov, 1968).

15 In questo tipo di relazione Komissarov identifica i seguenti tratti distintivi: non corrispondenza fra le rispettive strutture lessicali e sintattiche; marcata connessione di lessico e struttura testuale fra i due testi, mediante le necessarie trasformazioni semantiche e sintattiche; assenza di collegamenti logici tra gli enunciati dell'originale e gli enunciati della traduzione, indispensabili per stabilire la completa corrispondenza tra gli enunciati stessi; inferiore corrispondenza di contenuto fra originale e traduzione rispetto alle traduzioni considerate equivalenti. 
il contenuto derivato, non manifestamente espresso e tuttavia presente, che identifica, in realtà, l'autentica intenzione del testo, nonché il suo fine comunicativo $^{16}$.

Il secondo tipo di equivalenza è definito da Komissarov con l'aggettivo 'situazionale'17 e si differenzia dal primo in quanto si conserva, qui, un'integrazione dell'originale, cioè una descrizione della situazione, come riflesso, nel contenuto di un enunciato (e quindi del testo) di una situazione reale, mediante una delle possibili modalità descrittive di cui la lingua d'arrivo dispone. L'identicità della situazione descritta nei rispettivi e diversi testi è prova, osserva Komissarov, del carattere universale delle relazioni tra lingua e realtà extralinguistica ${ }^{18}$, esplicitato, a sua volta, nella fondamentale identicità semantica di testi linguisticamente diversi.

Il terzo tipo di equivalenza contempla sia la completa corrispondenza, tra i due testi considerati, della struttura del contenuto comunicato, sia l'impiego, nella traduzione, di costruzioni sinonimiche ${ }^{19}$.

16 Lo studioso, distinguendo fra «intenzione oggettiva», e concretamente realizzata, e «intenzione potenziale», sottolinea l'importanza del fine comunicativo, che rappresenta la parte più ampia di ogni enunciato e dell'insieme degli enunciati che costituiscono il testo. Con il termine 'fine', precisa Komissarov, non si intende né l'orientamento soggettivo e peculiare dell'emittente dell'enunciato (quindi del testo), né uno stimolo atto a suscitare una determinata reazione da parte del ricevente. Il fine della comunicazione, proprio come il restante contenuto dell'enunciato (e del testo) sussiste in seguito alla produzione dell'enunciato stesso in quanto dato oggettivo che riflette dunque non ciò che l'emittente intendeva affermare, bensì ciò che viene effettivamente affermato; il fine comunicativo non viene realizzato; esso si esprime nell'enunciato, e quindi nel testo.

17 È affine al primo tipo di equivalenza per via della mancata corrispondenza fra le componenti lessicali e strutturali dei rispettivi testi e, soprattutto, in virtù della conservazione, nel testo tradotto, del fine comunicativo, condizione necessaria dell'equivalenza. Inoltre viene qui mantenuto il riferimento alla stessa realtà referenziale del testo originale: l'insieme dei referenti correlati, quindi degli oggetti materiali e fenomenici, gli oggetti della vita psichica e della coscienza e le relazioni e i fatti di lingua.

18 Anche qui si fa riferimento alla capacità intuitiva del locutore (quindi del ricettore e anche del traduttore dell'enunciato): lo studioso sovietico osserva che nella maggior parte dei casi i locutori, che utilizzano intuitivamente una certa lingua, percepiscono la corrispondenza fra situazioni descritte nei modi più vari e recepite come narrazioni plurime di uno stesso avvenimento.

19 Ai tatti distintivi dei primi due tipi di equivalenza si aggiunge la conservazione, nel testo tradotto, dei concetti generali, per mezzo dei quali si realizza la descrizione rappresentata nel testo originale. La conservazione dei concetti generali è assicurata dalla possibilità di realizzare una parafrasi semantica del contenuto comunicato. 
Nel quarto tipo di equivalenza le strutture morfologiche e sintattiche utilizzate nel testo tradotto si pongono in relazione con le connessioni interne al testo di partenza mediante trasformazioni dirette o indirette. Il confronto fra originale e traduzione pone quindi in evidenza un rilevante - anche se non completo - parallelismo della componente lessicale (la maggior parte dei termini dell'originale presenta dei traducenti di significato analogo) e un rilevante grado di corrispondenza delle strutture sintattiche mediante trasformazione diretta o indiretta.

Il quinto tipo di equivalenza permette di rilevare la massima corrispondenza semantica possibile fra testi in lingue diverse. Essa è dunque caratterizzata da un elevato grado di parallelismo nella struttura del testo; dalla massima correlatività della componente lessicale; infine dalla conservazione di tutti gli elementi costitutivi del significato.

Ponendo a confronto la prospettiva teorico-pratica argomentata ed esemplificata da Komissarov con l'ottica proposta da Catford e l'approccio illustrato da Nida e, soprattutto, considerando i singoli contributi alla riflessione teorica sulla pratica traduttiva come un percorso in realtà unitario, ancorché composito e complesso - in quanto contrassegnato da apporti epistemologici culturalmente connotativi - orientato a comprendere, definire, quindi trattare il fenomeno della traduzione nel modo più completo e preciso possibile, nell'analisi del concetto di equivalenza strutturato da Komissarov si individua in primo luogo un radicale mutamento di angolazione. L'equivalenza, nelle sue varie declinazioni, viene osservata e, quindi, descritta. Essa non viene, dunque, normativamente determinata e imposta, in quanto tratto distintivo e consustanziale alla traduzione stessa che è appunto tale in quanto equivalente al testo originale. Il fenomeno dell'equivalenza può così essere indagato anche nelle sue realizzazioni più profonde e complesse, o addirittura implicite, quando determinate dall'intenzione comunicativa che conferisce all'atto traduttivo un marcato carattere inferenziale. In secondo luogo Komissarov osserva le varie e possibili realizzazioni dell'equivalenza considerando simultaneamente le componenti lessicale, morfologico-sintattica e semantica e rilevando l'essenziale importanza dei fattori situazionali, dai quali, soprattutto se culturalmente connotati, dipende la realizzazione stessa dell'equivalenza.

Di indubbio interesse è, comunque, l'approccio quantitativo suggerito da Catford. Anche Catford, in realtà, si affida a un metodo descrittivo, che appunto permette di riportare i dati osservati e di elaborarli. In Komissarov la natura descrittiva diviene, poi, il postulato dell'esistenza della stessa nozione di equivalenza.

L'articolata ripartizione dei tipi di equivalenza - formale e dinamica - esplicitata da Nida trova, infine, in Komissarov una completa sintesi che contempla non soltanto gli elementi linguistici formali, bensì anche le componenti contestuali verso le quali il linguista britannico aveva orientato 
il proprio sguardo, profondamente innovativo perché multidisciplinare e pluridirezonale.

Considerando i successivi sviluppi della ricerca traduttologica, è opportuno rilevare l'interesse di Komissarov per la Skopos-Theorie, introdotta negli anni Settanta da Hans Vermeer e illustrata nell'opera Grundlegung einer allgemeinen Translationstheorie (Reiss, Vermeer, 1984). Il fondamento di questa teoria è costituito dall'individuazione dello scopo, che orienta ogni atto traduttivo: è preminente la ragion d'essere della versione interlinguistica di un determinato testo a implicarne la traduzione, e l'equivalenza si realizzerà, semplicemente, nella costanza funzionale tra il testo in lingua di partenza e il testo in lingua d'arrivo. Nella trattazione del concetto di equivalenza Komissarov, come si è visto, attribuisce una fondamentale importanza all'intenzione, quindi al fine comunicativo, del testo, in alcuni casi latente, inespresso e dunque soltanto potenziale, ma comunque e necessariamente oggetto di trasposizione interlinguistica. Nella formulazione della Skopos-Theorie Komissarov riconosce il valore di un contributo innovativo, cui egli assegna il merito di aver conferito alla ricerca traduttologica una nuova direzione, contemplando, innanzi tutto, l'intero spettro delle funzioni espletate dal traduttore, attribuendo allo studioso il compito di descrivere i diversi obiettivi dell'attività traduttiva, infine evidenziando l'importanza e il prestigio del lavoro del traduttore. Alla luce della preminente e orientante importanza dello scopo o fine traduttivo il linguista sovietico rileva, soprattutto, la distinzione tra i concetti di adeguatezza ed equivalenza della traduzione. Se una traduzione adeguata è tale in quanto conforme allo scopo in origine prestabilito - e la nozione di adeguatezza attiene al processo traduttivo - l'equivalenza concerne la realizzazione finale del processo, quindi la traduzione, con il testo originale. Di conseguenza, conclude Komissarov, una traduzione può risultare equivalente, in quanto particolare risultato realizzato nell'adeguatezza della traduzione con il testo originale: una traduzione può risultare equivalente rispetto a un determinato scopo (Komissarov, 2011).

Un'ulteriore, sebbene più circoscritta, accezione teorico-pratica dell'equivalenza traduttiva, in un contesto di studi e ricerche coevo all'attività scientifica di Vilem Komissarov, è quella attribuita ai linguisti canadesi Vinay e Darbelnet, i quali, ispirati dai primi lavori dello studioso e traduttore sovietico Andrej Fëdorov, elaborarono, a partire dalla fine degli anni Cinquanta, un'ampia analisi stilistica e comparativa, ponendo a confronto testi in lingua inglese con testi in lingua francese, osservando gli elementi differenziali e, soprattutto, identificando le strategie e i procedimenti traduttivi adeguati. I due autori impiegarono il termine 'equivalenza' per designare i casi in cui le lingue dei testi considerati descrivono la stessa situazione con elementi stilistici o strutturali diversi, tra questi si evidenzia l'esempio della resa dei proverbi e delle espressioni idiomatiche. 
Dell'esposizione teorico-pratica dei due linguisti canadesi Komissarov sottolinea il valore del corpus di testi, documenti reali, sui quali viene condotta l'analisi traduttologica che, a sua volta, apre nuove prospettive per l'applicazione di singole teorie, alla comparazione di copie di lingue. Il linguista sovietico, tuttavia, osserva contestualmente che non sempre nella trattazione di Vinay e Darbelnet risulta nettamente definita la distinzione fra la teoria della traduzione e la linguistica contrastiva. Non sempre, dunque, appare evidente se le corrispondenze, quindi le realizzazioni dell'equivalenza, rilevate dagli studiosi, derivano dall'analisi di traduzioni reali o se tali esempi di equivalenza interlinguistica manifestano, semplicemente, un'identità funzionale.

\section{Bibliografia}

Auroux, S. (a cura di) (1990): Les notions philosophiques. Dictionnaire, II, t. 1. Paris: Presses Universitaires de France.

Avramov, V. (1966): O knige Ž. Mounena Teoretičeskie problemy perevoda. Tetradi perevodčika, pp. 103-111.

Catford, J. (1965): A Linguistic Theory of Translation. London: Oxford University Press.

Chazel, F. (1990): Équivalence fonctionnelle. In: Auroux, S. (a cura di), Les notions philosophiques. Dictionnaire, II, t. 1. Paris: Presses Universitaires de France.

Equivalence (1990): In: Auroux, S. (a cura di), Les notions philosophiques. Dictionnaire, II, t. 1. Paris: Presses Universitaires de France.

Holmes, J. (1988): Translated! Papers on Literary Translation and Translation Studies. Amsterdam: Rodopi.

Kelly, D. (2011): Equivalence. In: Baker, M., Saldanha, G. (a cura di), Routledge Encyclopedia of Translations Studies, pp. 96-99. London-New York: Routledge.

Koller, W. (1979): Einführung in die Übersetzungswissenschaft. Herdelberg-Wiesbaden: Quelle und Meyer.

Komissarov, V. (1968): Specifika perevodčeskich issledovanij. Tetradi perevodčika, pp. 3-8.

Komissarov, V. (2011): Sovremennoe perevodovedenie. Moskva: R. Valent.

Nida, E. (1964): Towards a Science of Translating: With Special Reference to Principles and Procedures Involved in Bible Translating. Leiden: E.J. Brill.

Procházka, V. (1942): Notes on Translating Technique. Slovoa Slovesnos. Journal of the Linguistic Circle of Prague, 8, pp. 1-20.

Rajchštejn, A. (1968): O perevode ustojčivych fraz. Tetradi perevodčika, pp. 29-43.

Reiss, K., Vermeer, H.J. (1984): Grundlegung einer allgemeinen Translationstheorie. Tübingen: Niemeyer.

Rieu, E.V., Phillips, J.B. (1955): Translating the Gospels. A Discussion between Dr. E. V. Rieu and the Rev. J. B. Phillips. Bible Translator, October, pp. 150-165. 